\title{
Produtos Florestais não Madeireiros do Brasil (2016-2020): Subsídio ao Estabelecimento de Novas Cadeias Produtivas pela Cooperativa de Extrativistas de Carajás
}

Luana do Carmi Oliveira Ferreira ${ }^{1}$, Gleysla Gonçalves de Carvalho Fernandes ${ }^{1}$, André Luís Macedo Vieira ${ }^{2} \&$ Álisson Rangel Albuquerque ${ }^{1}$

Recebido em 16/10/2020 - Aceito em 21/01/2021

${ }^{1}$ Universidade do Estado do Pará, Brasil. <luana.itups@gmail.com, gleysla14gg@gmail.com, alissonrangel@uepa.br >

${ }^{2}$ Instituto Chico Mendes de Conservação da Biodiversidade/ICMBio, Brasil. <andre.macedo@icmbio.gov.br>

\begin{abstract}
RESUMO - Este estudo expõe o manejo dos produtos florestais não madeireiros (PFNMs) como fator gerador de renda com o intuito de fortalecer e contribuir com a atividade extrativista realizada pela Cooperativa de Extrativistas de Carajás na Floresta Nacional do Tapirapé Aquiri (FLONATA). Para isso, realizou-se uma revisão sistemática com o auxílio da base de dados Web of Science e a partir dos filtros: data de publicação (2016 a 2020), palavra-chave "non-timber forest products" e origem (espécies brasileiras). Foram identificadas as principais espécies estudadas, o periódico de publicação, região de concentração das pesquisas, metodologia utilizada e seus principais usos com o objetivo de subsidiar o estabelecimento de novas cadeias produtivas dentro da FLONATA. Foram encontrados 564 estudos que abordavam aspectos relacionados aos produtos florestais não madeireiros no mundo, e desses, um total de 72 foram realizados no Brasil e apenas 56 apresentavam, prioritariamente, um uso não madeireiro para alguma espécie. Esses mesmos estudos propiciaram o levantamento de 16 famílias botânicas e 47 espécies com uso não madeireiro, onde 39 ocorrem naturalmente na Floresta Nacional do Tapirapé Aquiri, como é o caso da castanha-do-Pará (Bertholletia excelsa Bonpl.) e do açaí (Euterpe oleracea Mart). O levantamento dessas espécies com potencial de uso não madeireiro juntamente a especificidade de seus estudos como é o caso de informações ligadas a ecologia, fenologia, botânica entre outras áreas que caracterizam individualmente tal espécie, fomentam futuras possibilidades de uso contribuindo para o estabelecimento de novas cadeias produtivas e para a conservação da floresta.
\end{abstract}

Palavras-chave: Espécies não madeireiras; Floresta Nacional do Tapirapé Aquiri; produtos da floresta; revisão bibliográfica sistêmica.

\section{Non-Timber Forest Products of Brazil (2016-2020): Subsidy for the Establishment of New Productive Chains by the Carajás Extractivist Cooperative}

\begin{abstract}
This study exposes the management of non-timber forest products (NTFPs) as an income-generating factor in order to strengthen and contribute to the extractive activity carried out by the Carajás Extractive Cooperative in the Tapirapé Aquiri National Forest (FLONATA). For this, a systematic review was carried out with the help of the Web of Science database and from the filters: publication date (2016 to 2020), keyword "non-timber forest products" and origin (Brazilian species). The main species studied were identified, the publication periodical, the research concentration region, the methodology used and its main uses in order to support the establishment of new production chains within FLONATA. A total of 564 studies were found that addressed aspects related to nontimber forest products in the world, and of these, a total of 72 were carried out in Brazil and only 56 presented, primarily, a non-timber use for some species. These same studies provided a survey of 16 botanical families and 47 species with non-timber use, where 39 occur naturally in the Tapirapé Aquiri National Forest, such as Brazil nuts (Bertholletia excelsa Bonpl.) and açaí (Euterpe oleracea Mart). The survey of these species with potential for non-timber use, together with the specificity of their studies, such as information related to ecology, phenology, botany, among other areas that individually characterize this species, foster future use possibilities, contributing to the establishment of new chains productive activities and for forest conservation.
\end{abstract}

Keywords: Non-timber species; Tapirapé Aquiri National Forest; forest products; systemic bibliographic review. 


\title{
Productos Forestales no Maderables de Brasil (2016-2020): Subsidio para el Establecimiento de Nuevas Cadenas productivas por Parte de la Cooperativa Extractivista Carajás
}

\begin{abstract}
RESUMEN - Este estudio expone el manejo de productos forestales no maderables amazónicos con el fin de fortalecer y contribuir a la actividad extractiva que se realiza en áreas de Uso Sostenible, previsto en el Plan de Manejo para la recolección de productos forestales no maderables en el Bosque Nacional Tapirapé Aquiri. Para ello, se llevó a cabo una revisión sistemática con la ayuda de la base de datos Web Office Science y utilizando la palabra clave "nom-maderables productos forestales" para el análisis de publicaciones nacionales e internacionales, orientadas a diferentes temas que reportan la dinámica de estas. productos en los últimos cinco años. Se encontraron 564 estudios que abordaron aspectos relacionados con los productos forestales no maderables en el mundo, de estos, un total de $(n=72)$ se realizaron en Brasil y solo $(n=56)$ presentaron una especie potencialmente no maderable prioritaria. Estos mismos estudios proporcionaron, entre otros temas, el análisis del relevamiento de 16 familias botánicas, con énfasis en la familia Arecaceae $(n=19)$, Lecythidaceae $(n=12)$ y Meliaceae $(n=3)$ y, además, se encontró $(n=47)$ especies potencialmente no maderables, donde $(n=39)$ de ellas se encuentran en el Bosque Nacional Tapirapé Aquiri, como es el caso de las castañas de Brasil (Bertholletia excelsa Humb. \& Bonpl.) y açaí (Euterpe Oleracea Mart). Se concluye que el manejo de los productos forestales no maderables en la Amazonía se ha formado a lo largo de los años a partir de conocimientos empíricos provenientes de las comunidades que manejan estos productos y generan participación y análisis ecológicos, productivos y económicos con el sector académico y cooperativa. Y que la prospección de estas especies potencialmente no maderables junto con la especificidad de sus estudios fomentan futuras posibilidades de uso para las especies que se encuentran en el Bosque Nacional Tapirapé Aquiri.
\end{abstract}

Palabras clave: Especies no maderables; Bosque Nacional Tapirapé Aquiri; productos forestales; revisión sistemática.

\section{Introdução}

O bioma amazônico apresenta uma extensão territorial no Brasil de $4.199 .249 \mathrm{~km}^{2}$ de área (Prodes, 2020) e contempla também uma dimensão internacional em outros nove países sul americanos (Aragon, 2017). Abriga inúmeras espécies animais e vegetais que, juntas, formam a floresta tropical com a maior biodiversidade do mundo (Vieira et al., 2018). As espécies presentes nesse ambiente revelam a significativa importância do mesmo na promoção de serviços ecossistêmicos como a manutenção da biodiversidade, a reciclagem de água e a manutenção do solo e dos estoques de carbono que evitam o aquecimento global (Turetta et al., 2017; Fearnside, 2018; Rosa et al., 2020).

Em contrapartida, o desmatamento sobre a Floresta Amazônica avança continuamente através de ações antrópicas negativas que propiciam a alteração do ambiente e proporcionam diversos impactos ambientais (Abadias et al., 2020; Leles et al., 2020; Rossoni \& Morais, 2020). Para Silva et al. (2014) e Medeiros (2018), a falta de políticas públicas voltadas ao extrativismo, intensifica o desenvolvimento de outras atividades rurais como a agricultura de corte e queima ou a pecuária extensiva.
A coleta dos produtos florestais não madeireiros (PFNMs) constitui-se como uma possibilidade econômica viável de extração, que procura manter a estrutura e a funcionalidade da floresta (Balzon et al., 2004; Guerra et al., 2008; Elias \& Santos, 2016). Os PFNMs oriundos de recursos vegetais $e$ arbóreos apresentamse, principalmente, como fontes de alimento, forragem, fibras, medicamentos e cosméticos (Soares et al., 2008; Schindler et al., 2018). Na Amazônia, a utilização dos PFNMs é realizada por comunidades tradicionais que manejam a floresta a partir do seu conhecimento empírico, o que fomenta o extrativismo e integra cada o contexto cultural, social e econômico dos envolvidos (Pedrozo et al., 2011; Medeiros, 2018). O manuseio desses PFNMs exercido pelas comunidades da Amazônia proporciona também o acesso dos produtos a diferentes mercados.

Na floresta, nas feiras e nas cadeias de comercialização, a valorização dos PFNMs faz-se necessária para a realização do manejo florestal sustentável (Guerra et al., 2008; Lima et al., 2020). Assim, os PFNMs apresentamse como uma alternativa viável de utilização dos recursos naturais, no que diz respeito à conservação das florestas (Fachinello, 2010). 
Para Silva (2014), o manejo dos PFNMs de maneira racional considera principalmente aspectos ligados a regeneração natural da espécie fonte de produtos não madeireiros, possibilitando não apenas a conservação da biodiversidade, como também a segurança alimentar de comunidades que residem o entono das florestas.

Localizada no conjunto de áreas protegidas conhecido como "Mosaico de Carajás", a Floresta Nacional do Tapirapé Aquiri (FLONATA) é uma unidade de conservação de uso sustentável conforme o Sistema Nacional de Unidades de Conservação (SNUC) (Brasil, 2000), cujo plano de manejo prevê a utilização de seus PFNMs (Brasil, 2006). Atualmente a atividade de coletar os PFMNs é realizada pela Cooperativa de Extrativista de Carajás (COEX), que tem como principais produtos a folha-do-jaborandi (Pilocarpus macrophyllus Stapf ex Hom) e sementes nativas. A cooperativa é responsável não apenas por coletar, mas também por armazenar, beneficiar e comercializar estes produtos.

Este trabalho visa contribuir positivamente para a expansão das cadeias de comercialização da COEX, a partir do levantamento de espécies com potencial de uso de produtos não madeireiros mediante a análise de diversos estudos relacionados aos PFNMs na Amazônia, subsidiando futuramente a inserção de novos produtos florestais não madeireiros no comércio local e regional.

\section{Material e Métodos Objeto de estudo}

A Floresta Nacional do Tapirapé Aquiri possui uma área total aproximada de 190.000 ha e está localizada nos municípios de São Félix do Xingu e Marabá, no sudeste do Estado do Pará. A FLONATA localiza-se na "Serra de Carajás" e integra o conjunto de unidades de conservação formada pela Reserva Biológica de Tapirapé, a Área de Proteção Ambiental do Igarapé Gelado, a Floresta Nacional de Carajás, a Reserva Indígena Xikrin do Cateté, a Floresta Nacional de Itacaiúnas (Rolim et al., 2005) e o Parque Nacional dos Campos Ferruginosos (ICMBio, 2018). Além disso, a FLONATA abrange várias tipologias florestais, destacando-se a associação de Floresta Ombrófila Aberta Submontana com Palmeiras, Floresta Ombrófila Densa Submontana e Floresta Ombrófila Aluvial, que cobrem mais de $50 \%$ da FLONATA (ICMBio, 2006).
A coleta dos PFNMs ocorre entre o limite da Floresta Nacional de Carajás e da FLONATA. A Cooperativa dos Extrativistas da FLONA de Carajás (COEX), sediada no município de Parauapebas no sudeste do estado do Pará, foi formalizada juridicamente em 2010, mas o grupo começou as suas atividades, ainda de forma clandestina, no início da década de 1980. Atualmente possui 40 cooperados, colhendo e vendendo a folha-dejaborandi (Pilocarpus microphyllus) e sementes nativas como a fava-de-paca (Stryphnodendron pulcherrimum Willd. Hochr).

\section{Meta-análise}

Nesse estudo utilizou-se a revisão bibliográfica sistemática PRISMA (Preferred Reporting Items for Systematic Reviews and Meta-Analyses) descrita por Moher et al. (2009) e adaptada por Moher et al. (2015). A pesquisa foi realizada na base de dados Web of Science, escolhida devido à alta representatividade científica da mesma (Martins et al., 2013), utilizando 2 filtros automáticos: (a) Filtro 1 - ano de publicação (2016 a 2020) e (b) Filtro 2 - palavra-chave "non-tiber products forest. Posteriormente foram aplicados os filtros manuais: (c) Filtro 3 - PFNM citado nos artigos devem ter origem brasileira e (d) Filtro 4 - o artigo deve abordar e citar o nome de uma espécie ou um conjunto de informações sobre a dinâmica natural da espécie. Foram consideradas apenas publicações que levassem em conta produtos florestais não madeireiros de origem vegetal (Klauberg et al., 2016) e que não considerem a utilização da madeira extraída. Optou-se pelo levantamento das publicações somente dos últimos 5 anos, de forma a incluir produtos ainda em pesquisa e não comercializados além dos já consolidados na literatura.

As espécies citadas nos artigos filtrados foram comparadas com listas florísticas disponíveis no inventário florestal realizado pela empresa STCP (STCP, 2014) em 2014 na FLONATA em área de operação do projetoSalobo. Nesse inventário foram alocadas, sistematicamente, 52 parcelas com 13 hectares totais de área amostrada com identificação de 118 espécies arbórea. A identificação das espécies se deu com auxílio de parabotânicos, pesquisadores especialistas vinculados a herbários e com auxílio da literatura especializada. Os produtos florestais não madeireiros encontrados 
nos estudos foram classificados por categorias de uso em: alimentício, medicinal, matéria prima para composição de cosméticos e para a confecção de artesanatos entre outros (Balzon et al., 2004; Soares et al., 2008). Concomitante a isso, a parte utilizada que compõem os PFNMs é representada por flores, folhas, sementes, óleos, cascas raízes entre outras partes que caracterizam a usabilidade das espécies analisadas

Nos artigos buscou-se registrar: (a) quais revistas internacionais e nacionais estão publicando pesquisas sobre os produtos florestais não madeireiros?; (b) quais são as espécies com potencial de uso de produtos não madeireiros mais estudadas?; (c) quais são seus principais usos?; (d) quais aspectos de interesse são abordados nos estudos com os produtos florestais não madeireiros?; (e) quais produtos estão sendo mais pesquisados ou desenvolvidos por região (bioma) brasileira?; (f) onde os produtos estão sendo mais estudados (unidades de conservação, áreas rurais, comunidade tradicionais, etc.)?; e (g) quais metodologias estão sendo empregados para estudar os produtos florestais não madeireiros?

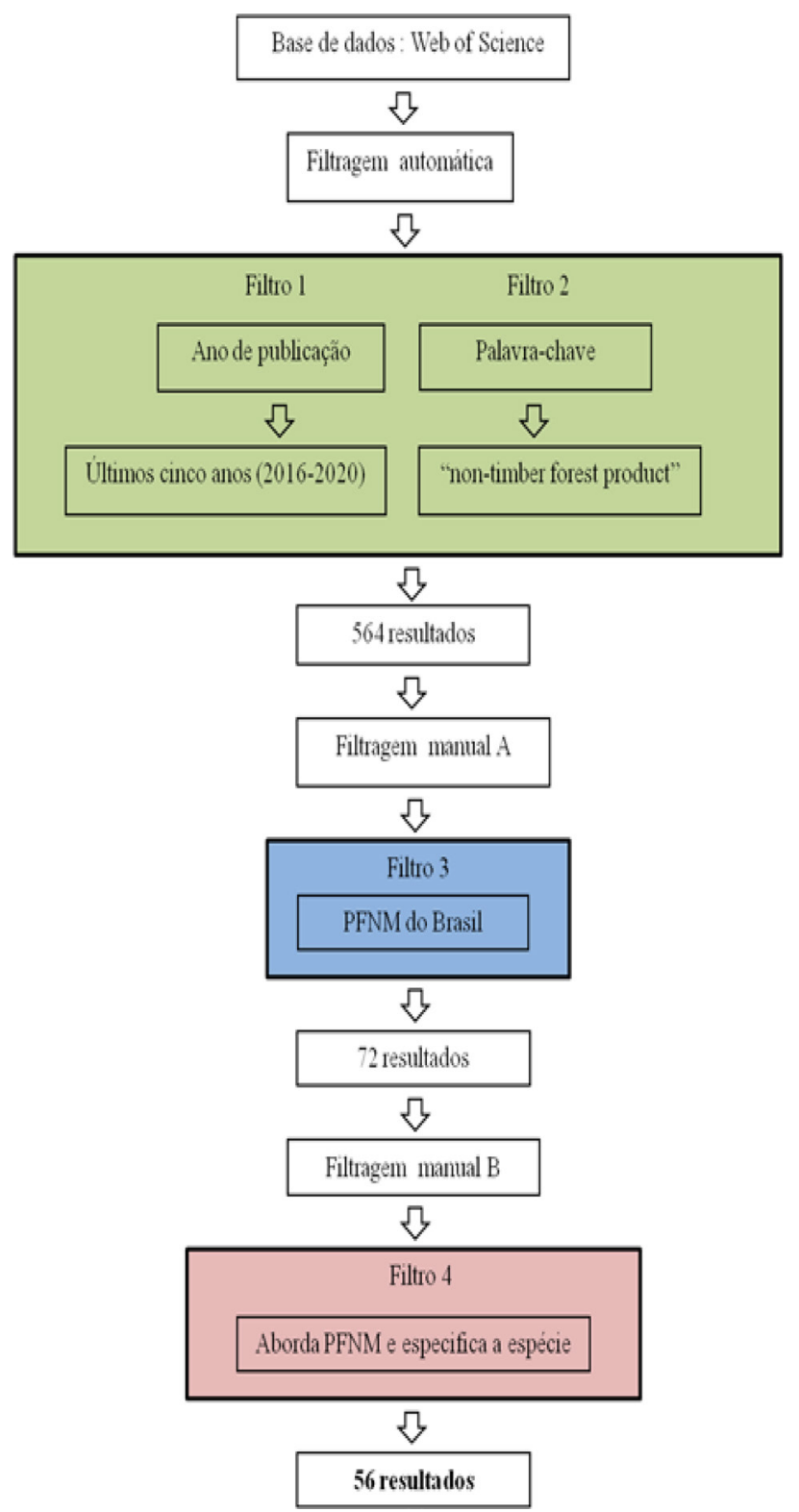

Figura 1 - Fluxograma das etapas de coleta de dados pelo Web of Science com aplicação dos filtros. 


\section{Resultados}

As publicações filtradas evidenciaram um número muito pequeno de publicações em revistas brasileiras, quando comparadas às publicações internacionais (Fig. 2-A e B). A Figura 2-A destaca a revista FOREST, com 26 estudos relacionados à dinâmica dos PFNMs em outros países e com apenas 2 estudos realizados no Brasil (Fig. 2-B). Em contrapartida, a revista NATIVA (Fig. 2-B) apresentou 20 estudos realizados no Brasil (Fig. 2-B) para 10 estudos sobre os produtos florestais não madeireiros desenvolvidos em outros países do mundo (Fig. 2-A).

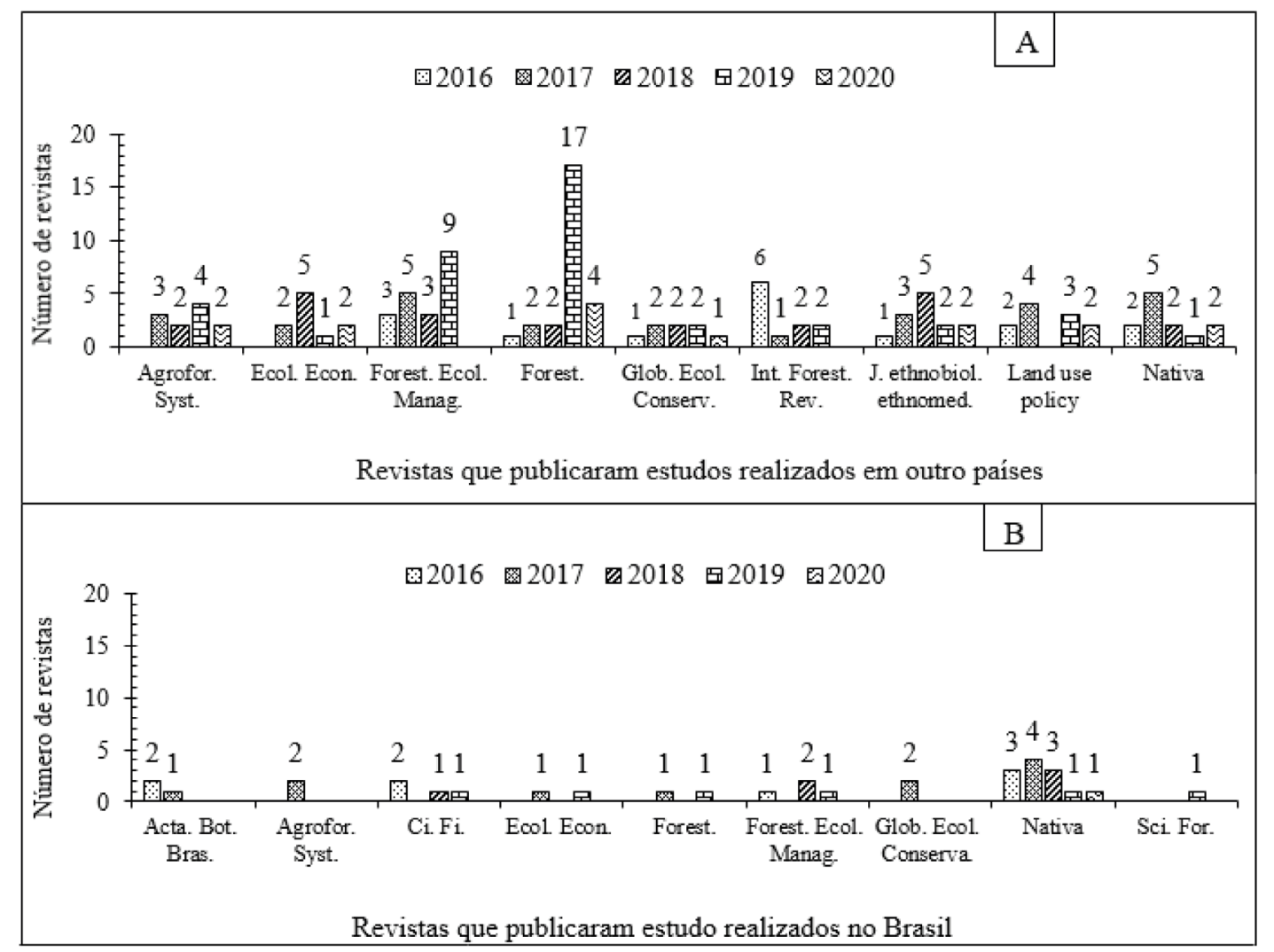

Figura 2 - Número de estudos sobre produtos florestais não madeireiros amazônicos publicados (2016 a 2020) de acordo com a base de dados Web of Science em: periódicos científicos internacionais (A) e periódicos nacionais $(B)$.

Periódicos internacionais (A) - Agrofor. Syst.: Agrorestry Systems, Ecol. Econ.: Ecologycal Economics, Foret. Ecol. Manag.: Forest Ecology and Management, Forest: Forest, Glob. Ecol. Conserv.: Global Ecology and Conservation, Int. Forest. Rev.: International Forest Review, J. ethnobiol. Ethnomed.: Jounal Of Etnobiology And Ethnomedicin, Land use policy: LAP: Land and Policy; Nativa: Nativa. Periódicos brasileiros (B) - Acta. Bot. Bra.: Acta Botanica Brasilica, Agrofor. Syst.: Agrorestry Systems, Ci. Fi.: Ciência Florestal, Ecol. Econ.: Ecologycal Economics; Forest.: Forest, Forest Ecology and Management.: Forest Ecology and Management; Glob. Ecol. Conserv.: Global Ecology and Conservation; Nativa.: Nativa.

O levantamento dos 56 estudos que abordavam os produtos não madeireiros no Brasil citou espécies pertencentes a 16 famílias botânicas, com destaque para: Arecaceae, com 19 espécies; Lecythidaceae, com 12 espécies e Meliaceae e Caryocariaceae, ambas com 3 espécies cada (Fig. 3-A). Também castanha-do-Pará (Bertholletia excelsa Bonpl.) (Batista et al., 2019) e o açaí e suas variedades como o Euterpe oleracea Mart. (Menezes et al., 2008) e Euterpe edulis Mart. (Lopes et al., 2019), ambos consumidos tradicionalmente na região amazônica (Fig. 3-B). 

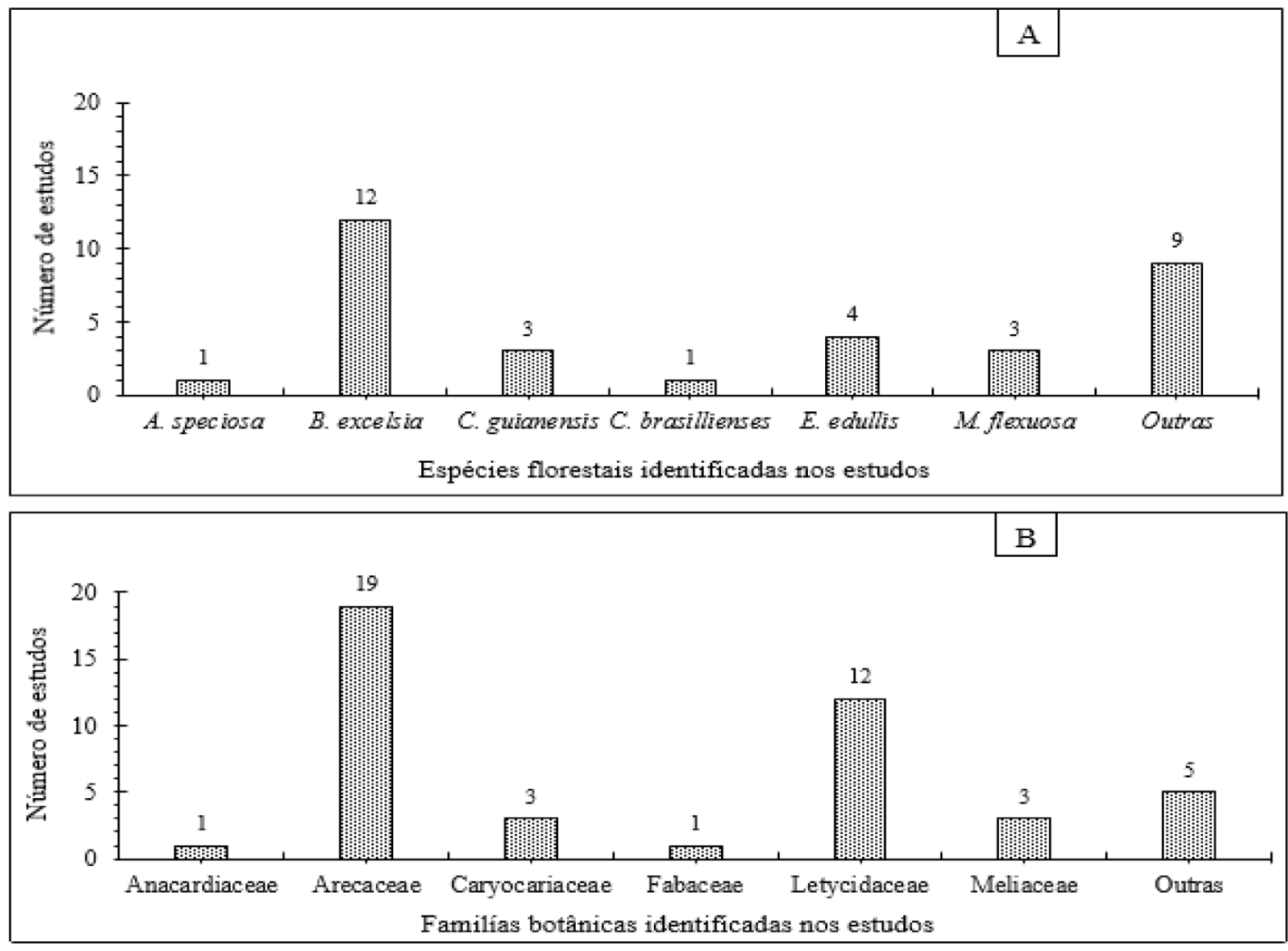

Figura 3-A - Número de espécies (A) e suas respectivas famílias botânicas (B) dos produtos florestais não madeireiros encontrados.

Espécies (A) - A. speciosa: Atallea speciosa; B. excelsa: Bertholletia excelsa; C. guianenses: Carapa guianenses; C. coriaceum: Caryocar coriaceum C. prunifera: Copenica prunifera; E. edullis: Euterpe edullis; E. precatoria: Euterpe precatoria; H. courbariul: Hymenea courbariul, M. flexuosa: Mauritia flexuosa.

Entre as publicações que abordam os PFNMs, a categoria mais estudada corresponde à alimentícia, com $33 \%$ dos produtos, seguindo da medicinal (9\%), cosmética (4\%) e artesanal (4\%). As mais utilizadas foram os frutos (30\%), óleos $(7 \%)$ sementes $(5 \%)$ e fibras $(3 \%)$.

Com a análise da distribuição geográfica dos estudos sobre PFNMs no Brasil, houve destaque para o bioma Amazônico com 35 publicações, seguidos pelos biomas Mata Atlântica e Cerrado. Na região amazônica, os estados do Pará, Amazonas e Acre foram aqueles que apresentaram maior número de publicações, com 9, 7 e 6 publicações, respectivamente (Fig. 4).

A partir dos resultados observados na Figura 5, observa-se que a área rural, caracterizada por regiões no perímetro rural das cidades $e$ de grandes centros urbanos, destacou-se na categoria local de realização da pesquisa/estudo, com um total de $34 \%$ trabalhos desenvolvidos. Majoritariamente, as pesquisas foram desenvolvidas em fazendas e atreladas a cooperativas e/ou associações ficando à frente das unidades de conservação federais (32\%). Os parques estaduais e municipais, plantios comerciais, reservas indígenas e feiras urbanas que comercializam PFNMs receberam menos citações.

Entre as metodologias aplicadas para a realização de pesquisas sobre PFNMs, as mais citadas foram o inventário florestal e aplicação de entrevistas semiestruturadas. Os inventários florestais permitem conhecer a diversidade e obter informações fitossociológicas das comunidades e populações da floresta (Gomes, 2019; Pinheiro et al., 2019; Pinto et al., 2019) e as entrevistas semiestruturadas permitem a obtenção de informações que descrevem a relação da coleta dos produtos não madeireiros com as pessoas envolvidas neste processo (Tonini et al., 2017; Lopes, 2019; Oliveira et al., 2019) (Fig. 6). 


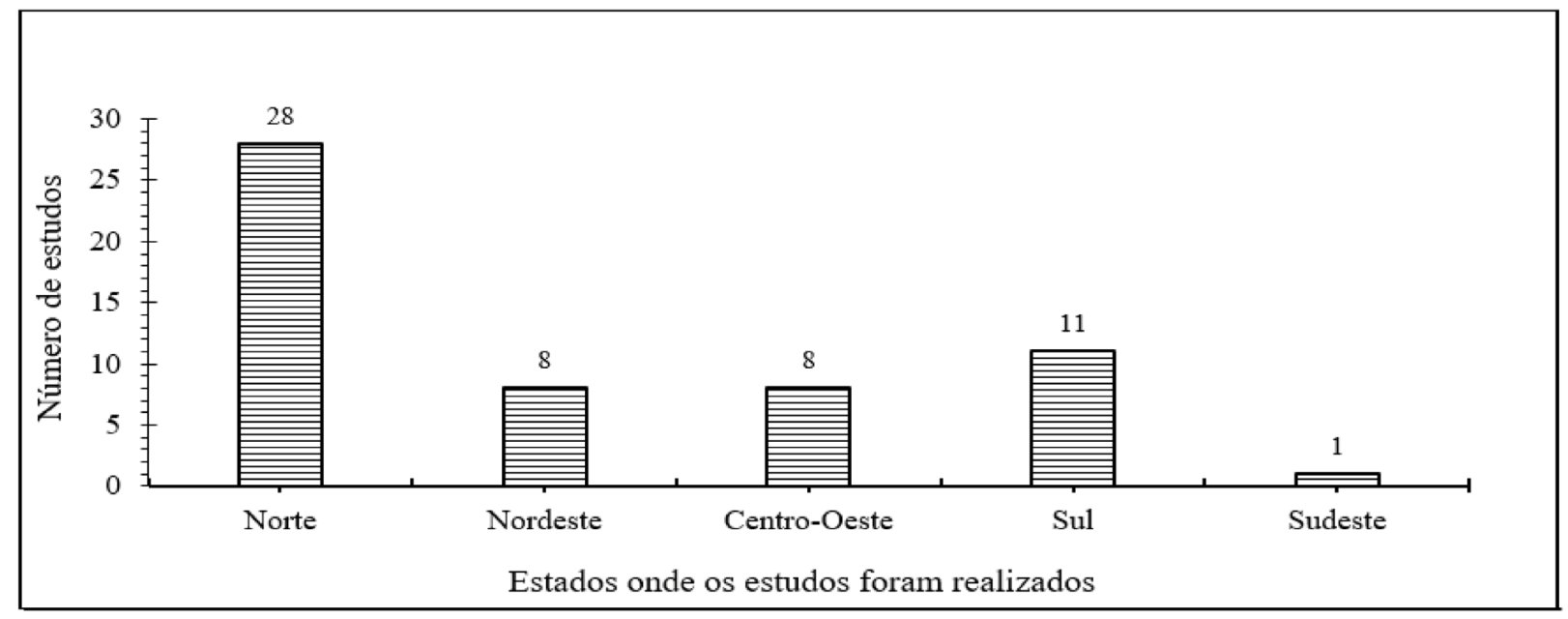

Figura 4 - Número de publicações (2016-2020) de produtos florestais não madeireiros, por região, no Brasil.

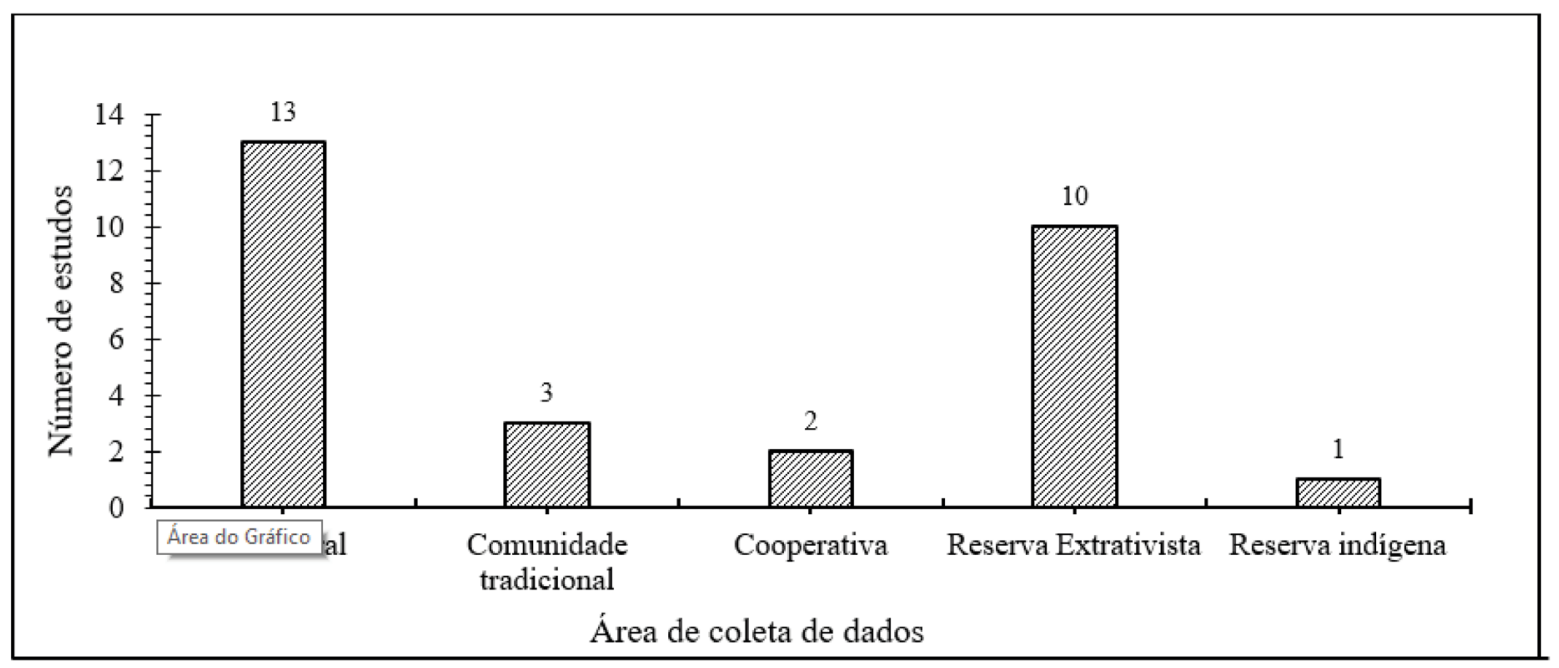

Figura 5 - Número de publicações (2016-2020) de produtos florestais não madeireiros, por local da pesquisa el ou coleta de dados.

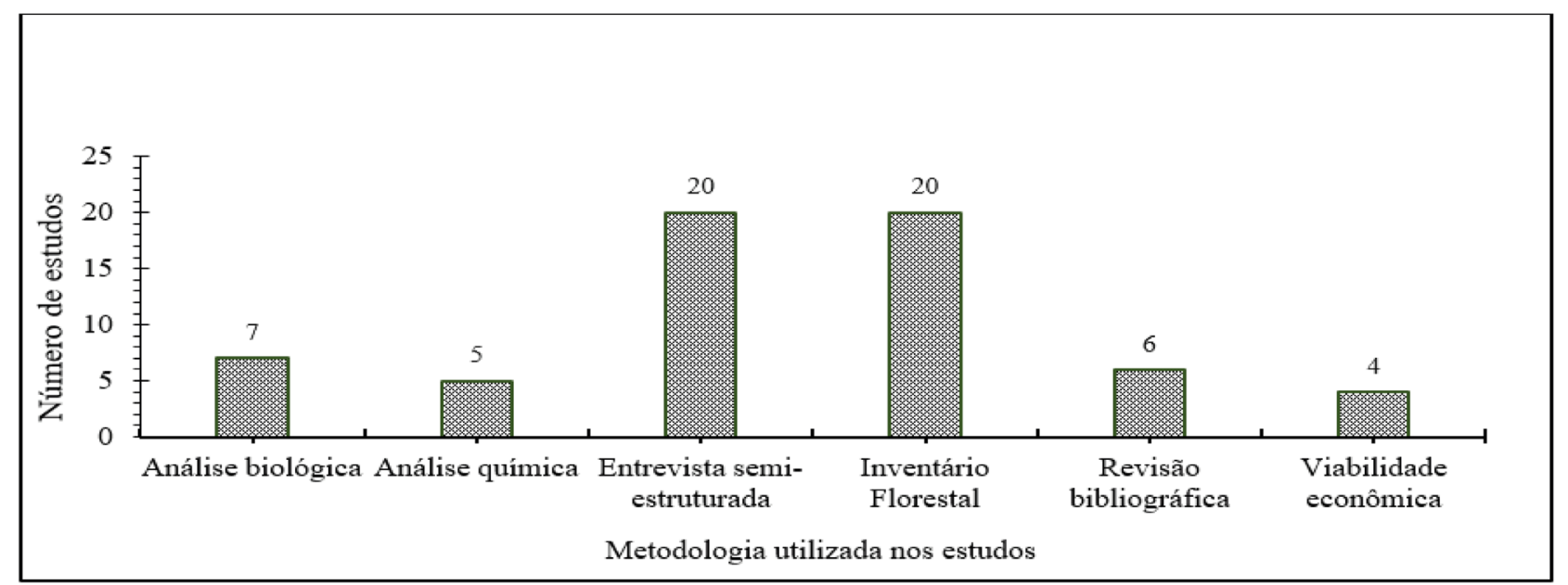

Figura 6 - Número de publicações (2016-2020) de produtos florestais não madeireiros em função da metodologia de coleta de dados. 
Nos estudos sobre os PFNMs, duas metodologias que envolvem biotecnologia foram citadas, as quais se referem à análise de biometria dos frutos da castanha-do-brasil (Bertholletia excelsa) aplicada por Borella et al. (2017) e à análise química para quantificação de pureza do óleo de copaíba (Copaifera spp. Ducke) proposta por Oliveira et al. (2017). Metodologias voltadas a revisões bibliográficas em prol do levantamento de informações sobre os PFNMs utilizados por Le Tourneau \& Beaufor (2016) ou à análise de viabilidade econômica para extração de produtos florestais não madeireiros, abordada nos estudos de Nakazono \& Magnusson (2016), tiveram menos citações dentre os trabalhos levantados.

A temática desses estudos, aliada ao objetivo e metodologia aplicada, abordava aspectos ligados à economia, com 20 estudos (Coelho, 2016; Vieira et al., 2016); à produção, com 9 estudos (Brum \& Souza, 2020; Elias \& Santos, 2016); ecologia, com 25 estudos (Le Tourneau \& Beaudor, 2017; Paludo et al., 2016); e ao histórico, com 5 estudos (Nakazono \& Magnusson, 2016). Entre as temáticas que menos foram citadas nos estudos analisados está a segurança do trabalho para os extrativistas, com apenas um estudo (Veiga et al., 2018).

\section{Discussão}

A biodiversidade brasileira apresenta um potencial real de utilização de recursos atrelada ao uso sustentável (Melo et al., 2010; Costa \& Simões, 2013; Medeiros, 2018). Os recursos extrativistas utilizados por meio de uma gestão sustentável $e$ ligados à Política de Garantia dos Preços Mínimos, como destaca Giatti et al. (2021), são alternativas viáveis para a conservação da biodiversidade $e$ geração de renda para as comunidades amazônicas. Entretanto, os PFNMs brasileiros efetivamente comercializados ainda se restringem a um grupo com poucas espécies, quando comparados ao número de espécies arbóreas e vegetais existentes.

A partir da análise dos estudos, os resultados apontam o bioma amazônico como um grande centro da diversidade arbóreo-vegetal brasileira; o que é conhecido, analisado e publicado por diversos pesquisadores como Vuola et al. (2019), Herrais et al. (2018), Moreira \& Ruhoff (2019). Essa rica diversidade biológica se estende por nove estados brasileiros, entre eles, o estado do Pará destaca-se como a unidade da federação onde foi realizado o maior número de estudos sobre espécies amazônicas; tais como os estudos realizados por: Vieira et al. (2016), sobre o uso do fruto buriti (Mauritia flexuosa L.f) e Cruz et al. (2017), sobre a utilidade do oléo de murumuru (Astrocarium murumuru Mart.).

Segundo o Idesp (2011) e Diniz et al. (2019), estudos referentes ao mercado local e regional de produtos florestais não madeireiros na região amazônica ainda são incipientes e suscetíveis a uma demanda do mercado. Para Nunes et al. (2011), a baixa rentabilidade e a sazonalidade são consideradas como grandes entraves para a consolidação econômica e social das comunidades extrativistas e, consequentemente, para a manutenção da floresta em pé. De acordo Klauberg et al. (2016), a valorização de novos PFNMs, ou seja, o subsídio à utilização de novas espécies fontes de produtos não madeireiros é essencial para o sucesso da gestão das cadeias de comercialização, para não ocorrer a superexploração de uma espécie.

$\mathrm{Na}$ Amazônia, o manejo dos PFNMs ainda é realizado da maneira tradicional por alguns extrativistas, ou seja, o manejo é realizado para subsistência da comunidade ou é destinado para o mercado local (Silva, 2014; Conceição et al., 2017; Lima et al., 2020). Logo, o manejo não madeireiro, juntamente ao conhecimento empírico de comunidades envolvidas com o extrativismo de PFNMS, deve considerar a ecologia da espécie, a fenologia, a produtividade, a densidade populacional, entre outros parâmetros que evidenciem o ciclo de vida de uma espécie ou de sua população, para que essas se tornem alternativas viáveis de promoção da sociobiodiversidade (Elias \& Santos, 2016; Brito, 2018).

A produção científica analisada evidenciou uma concentração das publicações com PFNMs oriundos de áreas rurais, devido à facilidade de acesso aos produtos. São exemplos os estudos realizados: no estado de Goiás, onde foram coletadas amostras do óleo de copaíba (Copaifera spp.) (Moreira, 2018) e outro realizado no estado Amapá, no qual foram coletados dados fenológicos relacionados a andiroba (Carapa guianenses) (Dantas et al., 2016).

Houve menção, também, de PFNMs oriundos de unidades de conservação federais, principalmente, em reservas extrativistas como a Reserva Extrativista Rio Cajari (Batista et al., 2019) e a Reserva Extrativista Médio do Juruá (Brites \& 
Morsello, 2018) e em florestas nacionais como a Floresta Nacional de Tapajós, Floresta Nacional do Tapirapé Aquiri e a Floresta Nacional do Amapá (com atuação de cooperativas extrativistas) (Gonçalves, 2012; Barbosa, 2017).

Na Floresta Nacional do Tapirapé Aquiri, a atividade extrativista é desenvolvida pela COEX que também realiza coleta na Floresta Nacional de Carajás (Benathar et al., 2020) e pelos agricultores do entono por meio do Programa Agroextrativista da FLONATA (Vieira et al., 2018).

Segundo Veríssimo et al. (2011), as áreas protegidas são ferramentas necessárias para a conservação da biodiversidade, pois as mesmas, segundo Vuola et al. (2018), viabilizam economicamente a utilização de PFNMs na geração de renda de pessoas que residem na área ou em seu entorno. Pinheiro et al. (2019), ao estudarem a fitossociologia em assentamento no estado do Pará, estimaram um valor monetário de US $\$ 2.539,84$ (ou $\mathrm{R} \$ 13.994,50$ ) para os PFNMs em uma área de 80 hectares. Ximenes et al. (2020) relatam, em seu estudo sobre a importância do açaí (Euterpe oleraceae) em uma comunidade quilombola no Estado do Pará, que a atividade gera renda familiar mensal estimada em US\$ 294,94 (ou $\mathrm{R} \$ 1.625,12$ ).

O subsídio a estudos que analisem $e$ fomentem o potencial não madeireiro de espécies nativas da Amazônia cujo uso ainda seja incipiente ou cuja cadeia produtiva ainda não seja estabelecida e difundida deve, segundo Silva et al. (2019), elencar o papel das políticas públicas para alavancar a atividade extrativista como alternativa à supressão vegetal. Nos estudos analisados, houve poucas menções voltadas às políticas públicas existentes, com destaque ao trabalho de Silva et al. (2016) que, ao analisarem as políticas públicas federais, encontraram um total de 19 programas relacionadas aos PFNMs, como é o caso da Política Geral de Preços Mínimos, instituída também para produtos extrativistas (Lima et al., 2017).

O manejo dos produtos florestais não madeireiros associados à comercialização deve, de acordo com Imperador \& Wart (2014) e Silva (2014), estar primordialmente ligado à certificação florestal, haja vista que oferece maior credibilidade às comunidades e empresas. De acordo com Imaflora (2009), os principais benefícios da certificação para o manejo florestal sustentável é a diferenciação do produto para o mercado consumidor, para o mercado consumidor, o que proporcionaria a inicialização de testes de comercialização de novos produtos $e$ a possibilidade de preços diferenciados.

\section{Conclusão}

Os resultados evidenciaram o atual cenário das pesquisas voltadas aos produtos florestais não madeireiros como alternativa para o manejo: pouco conhecimento de nossa biodiversidade $e$ de nossos produtos e um altíssimo potencial para exploração sustentável. Foram encontradas 47 espécies nativas com potencial de uso não madeireiro $e$, dessas, 39 também são encontradas na área da FLONATA; sendo apenas 2 com exploração de PFNMs em cadeia produtiva consolidada na UC: Bertholletia excelsa (catanhado-brasil) e Pilocarpus microphyllus (jaborandi). Este estudo tem caráter conservacionista, uma vez que reúne os dados das principais espécies brasileiras pesquisadas (entre 2016 a 2020) com potencial de uso não madeireiro, servindo como subsídio ao estabelecimento de novas cadeias de exploração sustentável na Floresta Nacional do Tapirapé Aquiri pela Cooperativa de Extrativistas de Carajás.

\section{Agradecimentos}

Ao ICMBio pelo concedimento da bolsa de Iniciação Científica e pela oportunidade para o desenvolvimento do projeto.

\section{Referências}

Abadias IM, da Fonseca PRB \& Barbos CH. Manejo da pecuária uma análise sobre impactos ambientais. Educa Amazônia-Educação, Sociedade e Meio Ambiente, 24 (1):113-125, 2020.

Aragón LE. A dimensão internacional da Amazônia: um aporte para sua interpretação/The international dimension of the Amazon: a contribution for its interpretation. Nera, (42): 14-33, 2018.

Balzon DR, Silva JCGL \& Santos AJ. Aspectos mercadológicos de produtos florestais não madeireiros: análise retrospectiva. Floresta, 34(3): 363-371, 2004.

Batista APB et al. Spatial association of fruit yield of Bertholletia excelsa Bonpl. trees in eastern Amazon. Forest Ecology and Management, 441, 99-105, 2019. 
Brasil. Lei $\mathrm{n}^{\circ}$ 9.985, de 18 de julho de 2000. Institui o Sistema Nacional de Unidades de Conservação da Natureza e dá outras providências. Publicada no Diário Oficial da União. Disponível em: <http://www. planalto.gov.br/ccivil_03/leis/L 9985.htm>. Acesso em 05/11/2016.

Brasil. Plano de Manejo Plano De Manejo Para Uso Múltiplo Da Floresta Nacional Do Tapirapé-Aquiri, IBAMA - Instituto Brasileiro do Meio Ambiente e dos Recursos Naturais Renováveis, 2006.

Benathar, ISC et al. 2020. Potential of Copaifera spp. oleoresin for sustainable extraction in the Eastern Amazon. Environment, Development and Sustainability, 1-13.

Borella DRS et al. Biometria de frutos e sementes da castanheira-do-Brasil da região Médio-Norte do Mato Grosso. Nativa, 5, 483-490, 2018.

Brites AB \& Morsello C. Effects of Economic Dependence and Cooperative Behavior Over Participation in Monitoring the Impacts of Natural Resource Trade. Ecological Economics, 147, 365-372, 2018.

Brito EJ. 2018. Produtos florestais não madeireiros vegetais e a subsistência da Comunidade Ribeirinha Paricatuba, município de Ponta de Pedras, Pará. TCC (Graduação em Engenharia Florestal) Universidade Federal Rural da Amazônia, Paragominas.

Brum HD \& Souza AF. Flood disturbance and shade stress shape the population structure of açaí palm Euterpe precatoria, the most abundant Amazon species. Botany, 98(3), 2020.

Coelho ML. Concentração regional do valor bruto de produção do pinheiro do paraná regional. Ciência Florestal, 26(3): 853-861, 2016.

Costa APD \& Simões AV. Extrativismo florestal nãomadeireiro do murumuru Astrocaryum murumuru Mart.: uma proposta de conservação do agroecossistema da comunidade de Santo Antônio, município de IgarapéMiri-Pará. Cadernos de Agroecologia, 8(2), 2013.

Conceição SP, Gama JRV, Monteiro RN, Ferreira RJS \& Souza OS. Cadeia produtiva do piquiá no município de Santarém, Estado do Pará, Brasil. Nativa, 5(1): 3136: 2017.

Elias GA \& Santos R. Produtos florestais não madeireiros e valor potencial de exploração sustentável da Floresta Atlântica no Sul de Santa Catarina. Ciência Florestal, 26(1): 249-262, 2016.

Dantas AR, Lira-Guedes AC, Mustin K, Aparício W, \&Guedes MC. Phenology of the multi-use tree species Carapa guianensis in a floodplain forest of the Amazon Estuary. Acta Botanica Brasilica, 30(4): 618-627, 2016.
Diniz MB, Diniz MJT, Ferreira AL \& Barrios MLC. Região Amazônica: biodiversidade e possibilidades de transformação industrial. Cadernos CEPEC, 6, 1-6, 2019.

Fachinello DT. 2010. Produtos Florestais NãoMadeiráveis (PFNM) no Estado de Rondônia e as Visões sobre Desenvolvimento e Sustentabilidade e Extrativismo. Dissertação (Mestrado em Administração) Universidade Federal de Rondônia, Porto Velho. 101p.

Fearside PM. The impact of land use on carbon stocks and fluxes: Implications for policy. Interactions between Biosphere, Atmosphere and Human Land Use in the Amazon Basin. Springer, 478p. 2016.

Giatti OF, Mariosa PH, Alfaia SS, Silva SCPD \& Pereira HDS. (2021). Potencial socioeconômico de produtos florestais não madeireiros na reserva de desenvolvimento sustentável do Uatumã, Amazonas. Revista de Economia e Sociologia Rural, 59, 2021.

Gomes CC, OMES \& Cristiano Cardoso. Potencial utilitário da vegetação lenhosa em área de Caatinga no estado de Pernambuco, nordeste do Brasil. Ciência Florestal, 29(1): 307-321, 2019.

Guerra FGPQ, Santos AJS, Sanquetta CR, Bittencourt \& AM Almeida AN. Quantificação e valoração de produtos florestais não madeireiros. Floresta, 39(2): 431-439, 2009.

Herraiz AD, Aurelio D, Alencastro G, Lima PM \& Fearnside PM. Amazonian flood impacts on managed Brazilnut stands along Brazil's Madeira River: A sustainable forest management system threatened by climate change. Forest ecology and management, 406, 46-52, 2017.

Homma AKO. 2008 Extrativismo, biodiversidade e biopirataria na Amazônia. Brasília, DF: Embrapa Informação Tecnológica.

ICMBio. 2006. (Instituto Chico Mendes de Conservação da Biodiversidade). Disponível em: > http://www.icmbio. gov.br/portal/images/stories/imgsunidadescoservacao/ flona_tapirape-aquiri.pdf. Acesso em: 01/01/2020

ICMBio. 2018. (Instituto Chico Mendes de Conservação da Biodiversidade). Disponível em:>https://www.gov. br/icmbio/ptbr/acessoainformacao/pesquisa/.<Acesso em 17 Dez 2020.

Imbeau LM, Pétry F \& Lamari M. Left-right party ideology and government policies: A meta-analysis. European Journal of Political Research, 40(1): 1-29, 2001.

Idesp. (Instituto de Desenvolvimento Econômico, Social e Ambiental do Pará). 2011. Cadeias de comercialização de produtos florestais não madeireiros na Região de integração Xingu, Estado do Pará. Relatório técnico, 2011. 193p. 
Imaflora. 2009. (Instituto de Manejo e Certificação Florestal e Agrícola). Disponível em:> http:/www. abihpec.org.br/conteudo/s02/cont/apresent_imaflora. ppt $<$.Acessoem01/01/2020.

Imperador AM \& Wadt LHO. Certificação de produtos florestais não madeireiros na perspectiva mercadológica de associações extrativistas no estado do acre. Holos, 1 , 126-135, 2014.

Klauberg C, Silva CA, Lima MP \& Carvalho SPC. Panorama mundial sobre publicações técnico-científicas abordando produtos florestais não madeireiros nas duas últimas décadas. Advances in Forestry Science, 3 (2): 29-37, 2016.

Krainovic P, Almeida D \& Sampaio P. New allometric equations to support sustainable plantation management of rosewood (Aniba rosaeodora Ducke) in the Central Amazon. Forests, 8(9): 327, 2017.

Leles W, Davis J, Ribeiro A \& Filho BSS. Amazônia do futuro: o que esperar dos impactos socioambientais da Ferrogrão?. Policy Brief, 2020. Disponível em:> Ferrograo_policy-brief_.pdf (ufmg.br) <. Acesso em 03/07/2021

Le Tourneau FM \& Beaufort B. Exploring the boundaries between individual and collective land use management in a CPR system: the PAE Chico Mendes (Acre, Brazil). International Journal of the Commons, 11(1), 2017.

Lima $\mathrm{RB}$ et al. Valoração de componentes não madeireiros na Amazônia: metodologias de quantificação para a geração de renda. Brazilian Applied Science Review, 4(2): 561-591, 2020.

Lima CVS, Júnior HMC \& Lunas DAL. A política de garantia de preços mínimos para produtos da sociobiodiversidade (PGPM-Bio): potencialidades da intervenção estatal para a preservação ambiental em Goiás. Guaju, 3 (1): 37-65, 2017.

Lopes E, Soares FB, Rajão R, Merry F \& Ribeiro SC. Mapping the socio-ecology of Non Timber Forest Products (NTFP) extraction in the Brazilian Amazon: The case of açaí (Euterpe precatoria Mart) in Acre. Landscape and Urban Planning, 188, 110-117, 2020.

Lopes HS, 2019. Caracterização florística e estrutural em fitofisionomia de capão na savana metalófila na Flona Carajás-PA. TCC (Bacharelado em Engenharia Florestal) - Universidade Federal Rural da Amazônia, Parauapebas.

Maciel RCG, Reydon BP, Costa JAD \& Sales GDODO. Pagando pelos serviços ambientais: uma proposta para a Reserva Extrativista Chico Mendes. Acta Amazônica, 40(3): 489-498, 2010.
Martins AI, Queirós A, Rocha NP \& Santos, BS. Avaliação de usabilidade: uma revisão sistemática da literatura. Revista Ibérica de Sistemas e Tecnologias de Informação, (11): 31, 2013.

Melo ACS, Moreira BB \& Alencar A. Análise de desemprenho logístico das cadeias produtivas de produtos florestais não madeireiros oriundos da região amazônica. Traços, 12(26): 27-37, 2010.

Moher D et al. Preferred reporting items for systematic review and meta-analysis protocols (PRISMA-P) 2015 statement. Systematic reviews, 4(1), 1-9, 2015.

Moher D, Tetzlanf J, Trico AC, Sampsom M \& Altman DG. Epidemiology and reporting characteristics of systematic reviews. Plos Med, 4(3), 2009.

Menezes EMS, Tores Amanda T \& Sabaa SAU. Valor nutricional da polpa de açaí (Euterpe oleracea Mart) liofilizada. Acta amazônica, 38(2): 311-316, 2008.

Moreira AA \& Ruhoff AL. 2019. Padrões de evapotranspiração na Amazônia. In Anais Simpósio Brasileiro de Recursos Hídricos. Porto Alegre: ABRH. 23p.

Moreira ACO. Espectroscopia NIR, CG-EM e quimiometria para o controle de qualidade do óleo de copaíba (Copaifera spp.). Tese (Doutorado em Tecnologias Química e Biológica) Universidade de Brasília, Brasília, Distrito Federal, 2018.

Nakazono EM \& Magnusson WE. Unsustainable Management of Arumã (Ischnosiphon polyphyllus [Poepp. \& Endl.] Körn.) by the Novo Airão Artisans Association, Rio Negro, Amazon, Brazil. Economic Botany, 70(2), 2016.

Nunes FSM, Soares FBS \& Rodrigues H. 2011. Valorando a floresta em pé: rentabilidade da castanha do Brasil no Acre. In Anais: IX Encontro Nacional da Sociedade Brasileira de Economia Ecológica. Brasília.

Oliveira AS et al. Bringing economic development for whom? An exploratory study of the impact of the Interoceanic Highway on the livelihood of smallholders in the Amazon. Landscape and Urban Planning, 188, 171-17, 2019.

Oliveira ACM, Machado AHL, de Almeida, FV \& Braga JWB. Rapid purity determination of copaiba oils by a portable NIR spectrometer and PLSR. Food Analytical Methods, 11(7): 1867-1877, 2018.

Paludo GF, Duarte RI, Bernardi, AP, Mantovi A \& Reis MSR. The size of Araucaria angustifolia (Bertol.) Kuntze entering into reproductive stages as a basis for seed management projects. Revista Árvore, 40 (4), 2016.

Predozo EA, Silva TN, Sato SAS \& Oliveira NDA. Produtos Florestais Não Madeiráveis (PFNMS): as Filières do Açaí e da Castanha da Amazônia. Revista de Administração e Negócios da Amazônia-RARA, 3(2): 88-112, 2011. 
Plese NGSP \& Perreira, HF. Efetividade local das políticas públicas estaduais de fomento à coleta sustentável de produtos florestais não-madeireiros: o caso da Reserva Extrativista do Cazumbá, no Acre, Brasil. Brazilian Journal of Development, 6(9) 7273172748, 2020.

Pinheiro JC et al. Fitossociologia e expectativa de renda com produtos florestais madeireiros e não madeireiros em assentamento no Pará. Nativa, 7(1): 101-108, 2019.

Pinto LCL, Rodrigues IPS \& Drumond MA. Population Structure and Fruit Productivity Analyses in Support of the Use of Caryocar brasiliense. Floresta e Ambiente, 26(2), 2019.

Prodes (Monitoramento do Desmatamento da Floresta Amazônica Brasileira por Satélite). Disponível em:> http://www.obt.inpe.br/OBT/assuntos/programas/ amazonia/prodes < . Acesso em: 22/09/2020.

Rosa JCS, Souza BA \& Sánchez LE. Identificação de serviços ecossistêmicos em áreas de floresta mediante sensoriamento remoto. Revista Desenvolvimento e Meio Ambiente, 53: 276-295, 2020.

Ribeiro SMC et al. Can multifunctional livelihoods including recreational ecosystem services (RES) and non-timber forest products (NTFP) maintain biodiverse forests in the Brazilian Amazon? Ecosystem Services, 31, 517-526, 2018.

Ribeiro ALP \& Souza MR. Revisão sistemática e metaanálise de estudos de diagnóstico e prognóstico: um tutorial. Arquivos Brasileiros de Cardiologia, 92(3), 2009.

Rolim SG, Couto HTZ, Jesus RM \& França JT. Modelos volumétricos para a Floresta Nacional do Tapirapé-Aquirí, Serra dos Carajás (PA). Acta Amazônica, 36(1), 2006.

Rossoni RA \& de Moraes ML. Agropecuária e desmatamento na amazônia legal brasileira: uma análise espacial entre 2007 e 2017. Geografia em Questão, 13(3), 2020.

Sampaio RF \& Mancini MC. Estudos de revisão sistemática: um guia para síntese criteriosa da evidência científica. Revista Brasileira de Fisioterapia, 11(1), 2007.

Santiago HPC, Mazalli MR, Rodrigues CEC \& Oliveira AL. Extraction of Brazil nut kernel oil using green solvents: Effects of the process variables in the oil yield and composition. Journal of Food Process Engineering, 42(7)13271, 2019.

Silva CK. Potencial produtivo e manejo de dois produtos florestais não madeireiros no contexto Amazônico - o cipó-titica (Heteropsis spp.) e o óleo de copaîba (Copaifera spp). Tese (Doutorado em Recursos Florestais), Universidade de São Paulo, Piracicaba, 2014.
Silva M, Martins B, Nagy A \& Maciel N. Políticas públicas do Brasil para produtos florestais não madeireiros. The Overarching Issues of the European Space- a strategic (re)positioning of environmental and socio-economic. Faculdade Letras Univ. Porto. 255-273, 2016.

Silva ER, Mendonça AR, Fernandes MM, Dias HM \& Silva MLM. Produtos florestais não madeireiros e valoração ambiental da Floresta Nacional de Pacotuba, ES. Revista de Ciências Agroveterinárias, 18(3), 363373, 2019.

Soares TS, Fiedler NC, Silva JÁ \& Gasparini AJ. Produtos Florestais Não Madeireiros. Revista Científica Eletrônica de Engenharia Florestal, 11: 7, 2008.

Souza FF, Silva CVS \& Barros FB. The invisible market of miriti (Mauritia flexuosa L.f.) fruits, the "winter acai", in Amazonian riverine communities of Abaetetuba, Northern Brazil. Global Ecology and Conservation, 14: 00393, 2018.

STCP (STCP Engenharia de Projetos Ltda). Inventário Florestal Realizado em uma área de 408, 47 hectares destinados à fase de operação do projeto Salobo, Floresta Nacional do Tapirapé Aquiri. Relatório técnico, 2014.

Tonini H, Baldoni AB, Hoogerheide ES S \& Botelho SDCC. Caracterização e rentabilidade do sistema extrativista da castanha-do-brasil praticado em Itaúba (MT). Nativa, 5(3), 175-181, 2017.

Veríssimo A et al. Áreas Protegidas na Amazônia Brasileira: avanços e desafios Belém/São Paulo: Imazon e ISA, 2011.

Veiga JPC, Trevisani DM, Makishi F, Abreu MGCD, Silva MSP \& Zacareli MA. Health and safety standards in labor and extractivism: the case of rural communities in Brazilian Amazon. Saúde e Sociedade, 26(3): 774$785,2017$.

Vieira IR, Oliveira JS. Santos KP, Silva GO, Vieira FJ \& Barros, RA. Contingent valuation study of buriti (Mauritia flexuosa Lf) in the main region of production in Brazil: is environmental conservation a collective responsibility?. Acta Botanica Brasilica, 30(4): 532539, 2018.

Vieira ALM, Mendonça MV, Albuquerque SDS, Bezerra JR \& Silva RGD. Práticas agroecológicas no entorno da Floresta Nacional do Tapirapé-Aquiri. Cadernos de Agroecologia, 13(1), 2018.

Vuola M, Bauch SC \& Sills EO. The regional market for non-timber forest products. Desenvolvimento e Meio Ambiente, 48, 2018.

Ximenes LC, Gama JRV, Bezerra TG \& Cruz SG. Importância do açaí na renda mensal da comunidade quilombola Murumuru em Santarém, Pará. Revista Ibero-Americana de Ciências Ambientais, 11(2), 3642, 2020. 
Biodiversidade Brasileira - BioBrasil.

Edição Temática: PIBIC

n. 1,2022

http://www.icmbio.gov.br/revistaeletronica/index.php/BioBR

Biodiversidade Brasileira é uma publicação eletrônica científica do Instituto Chico Mendes de

Conservação da Biodiversidade (ICMBio) que tem como objetivo fomentar a discussão e a disseminação de experiências em conservação e manejo, com foco em unidades de conservação $e$ espécies ameaçadas.

ISSN: 2236-2886 